\title{
Effects of Riboflavin and Selenium Deficiencies on Glutathione and Its Relating Enzyme Activities with Respect to Lipid Peroxide Content of Rat Livers
}

\author{
Misako TANIGUCHI and Takayuki HARA ${ }^{1}$ \\ Department of Food and Nutrition, Nakamura Gakuen College, \\ 5-7-1 Befu, Jyonan-ku, Fukuoka 814, Japan
}

(Received August 11, 1982)

\begin{abstract}
Summary Weanling male rats were fed a riboflavin- or seleniumdeficient diet for 5 weeks, and the glutathione content and its relating enzyme activities in the livers were examined. The glutathione content and glutathione reductase activity were decreased by deficiency of riboflavin, but not by that of selenium. Glutathione peroxidase activity was increased by addition of selenium to the diet, but without its addition, the activity was higher in the riboflavin-deficient rats than in the riboflavin-sufficient rats, in spite of the increase of lipid peroxides in the former rats. Glucose 6-phosphate dehydrogenase activity was decreased significantly by riboflavin deficiency.

In another experiment, riboflavin was intraperitoneally injected into rats fed the diet to which neither riboflavin nor selenium had been added. The glutathione content and activities of glutathione reductase and glucose 6phosphate dehydrogenase returned to the control level of riboflavinsufficient rats in $24 \mathrm{~h}$, the lipid peroxide level in $48 \mathrm{~h}$, and the glutathione peroxidase activity, being higher than that in the control rats, in $72 \mathrm{~h}$ after the injection, respectively.

These findings indicate that the increase of lipid peroxides in the livers of riboflavin-deficient rats is caused by the decrease in the glutathione content as well as glutathione reductase activity rather than by that in the selenium-dependent glutathione peroxidase activity.

Key Words riboflavin and selenium deficiencies, riboflavin deficiency and glutathione, riboflavin deficiency and lipid peroxides, glutathione peroxidase and lipid peroxides
\end{abstract}

Increase of lipid peroxides was observed in the livers and serum of rats which were fed a riboflavin-deficient diet (1). Much research has been extensively made to clarify the mechanism of lipid peroxidation, and several investigators have de-

\footnotetext{
1 谷口巴佐子, 原 孝之
} 
monstrated that NADPH-cytochrome P-450 reductase is involved in NADPHdependent lipid peroxidation $(2,3)$. In order to resolve the mechanism of increase of lipid peroxides in the rats which were fed the riboflavin-deficient diet (riboflavindeficient rats hereafter), the authors studied NADPH-dependent lipid peroxidation with liver microsomes and found that the formation of lipid peroxides expressed as malondialdehyde was decreased in the deficient rats, instead of an increase of lipid peroxide content in the livers.

The increase of lipid peroxides in the livers may be accounted for by several reasons; (i) disturbance and modification of electron transferring systems in NADPH-cytochrome P-450 reductase or NADPH-cytochrome $b$ reductase, (ii) diminution of capacity to degradate hydrogen peroxide which is successively transformed into $\mathrm{OH}^{*}$ by Fenton-type or Harber-Weiss reaction, (iii) decrease in removal of organic peroxides, and consequent acceleration in the propagative step of lipid peroxidation as suggested by Svingen et al.(4), etc. On the other hand, if antioxidants or other reducing substances are lost during the preparation of microsomes, the in vitro lipid peroxidation with microsomes does not reflect the in vivo state of that in the intact livers.

In our study, the existence of abnormal types of NADPH-cytochrome P-450 reductase was found in the liver microsomes of riboflavin-deficient rats, which consists of $2 \mathrm{~mol}$ of FAD instead of $1 \mathrm{~mol}$ each of FMN and FAD per mole of the enzyme (5). Vermilion and Coon demonstrated that the FMN-depleted enzyme prepared from the native enzyme lost the ability to transfer electrons to cytochrome P-450 (6). Therefore, it seems likely that the disturbance of electron transfer occurs in the liver microsomes of riboflavin-deficient rats, and accelerates lipid peroxidation.

Glutathione peroxidase which protects biological systems against lipid peroxidation has been shown to require specifically glutathione as the reductant (7). Glutathione reductase is a flavoenzyme and participates in maintaining the glutathione concentration of the reduced form in cells. In the present paper, glutathione content and activities of glutathione reductase and peroxidase in the riboflavin-deficient rat livers were examined to clarify the inconsistent results of a decrease of lipid peroxidation activity and an increase of lipid peroxides in the livers.

\section{EXPERIMENTAL}

1. Animals and diets. Weanling male rats of the Wistar strain were fed the riboflavin-deficient diet for 5 weeks as described in our previous paper (1). The control rats were fed a diet to which $40 \mathrm{mg}$ of riboflavin per $\mathrm{kg}$ of the deficient diet had been added. Diets and distilled water were given ad libitum. In a recovery experiment, $50 \mathrm{mg}$ of riboflavin per $\mathrm{kg}$ of body weight was intraperitoneally injected into deficient rats daily. In another experiment, rats were fed the riboflavin-deficient or supplemented diet to which $0.5 \mathrm{ppm}$ selenium in the form of sodium selenite was 
added. The selenium content in vitamin free casein (ICN Nutritional Biochemicals, Cleaveland, Ohio, U.S.A., Lot No. 7433), mineral mixture, and the riboflavindeficient or supplemented diet were determined by the method of Olson et al. (8), and $0.15,0.015$ and $0.034 \mathrm{ppm}$ selenium were found respectively. The seleniumsupplemented diet contained $0.534 \mathrm{ppm}$ selenium.

2. Assay of enzymatic activities. The livers were immediately removed and homogenized in 10 volumes of $0.25 \mathrm{M}$ sucrose solution $\left(0-4^{\circ} \mathrm{C}\right)$ containing $1 \mathrm{mM}$ Tris- $\mathrm{HCl}(\mathrm{pH} 7.4)$ and $0.1 \mathrm{~mm}$ EDTA. The homogenates were centrifuged at $10,000 \times g$ for $20 \mathrm{~min}$. The postmitochondrial supernatant was centrifuged at $100,000 \times g$ for $60 \mathrm{~min}$, and the supernatant was used for assay of enzymic activities.

Glutathione peroxidase activity was assayed by the method of Tappel( 9 ), and the rate of reduction in absorbance at $340 \mathrm{~nm}$ was measured at $37^{\circ} \mathrm{C}$. The reaction mixture of $1.5 \mathrm{ml}$ contained: $50 \mathrm{mM}$ Tris- $\mathrm{HCl}$ ( $\mathrm{pH} 7.6$ ), $0.1 \mathrm{mM}$ EDTA, $0.25 \mathrm{~mm}$ glutathione (oxidized form), $0.12 \mathrm{~mm}$ NADPH and 1 unit of glutathione reductase. The reaction was initiated by addition of $0.05 \mathrm{ml}$ of cumene hydroperoxide $(1 \mathrm{mg} / \mathrm{ml})$. Glutathione reductase activity was measured by the method of Carlberg and Mannervik (10). The reaction mixture of $3.0 \mathrm{ml}$ contained: $0.1 \mathrm{M}$ sodium phosphate buffer ( $\mathrm{pH} 7.6$ ), $0.5 \mathrm{~mm}$ EDTA, $1 \mathrm{~mm}$ glutathione (oxidized form) and $0.1 \mathrm{mM}$ NADPH. The rate of reduction in absorbance at $340 \mathrm{~nm}$ was measured at $30^{\circ} \mathrm{C}$. Glucose 6-phosphate dehydrogenase activity was determined at $25^{\circ} \mathrm{C}$ by measuring the reduction of NADP at $340 \mathrm{~nm}$. The reaction mixture of $3 \mathrm{ml}$ contained; $0.1 \mathrm{M}$ Tris- $\mathrm{HCl}(\mathrm{pH} 7.6), 10 \mathrm{mM} \mathrm{MgCl}, 2 \mathrm{~mm}$ glucose 6-phosphate and $1.2 \mathrm{~mm}$ NADP.

One enzyme unit was expressed as the amount of enzyme that oxidized $1 \mu \mathrm{mol}$ of NADPH in glutathione peroxidase and reductase or reduced that of NADP in glucose 6-phosphate dehydrogenase, respectively. The specific activity was expressed as units per mg of protein.

3. Assays of glutathione and lipid peroxides. A portion of livers $(0.1$ to $0.2 \mathrm{~g})$ was quickly homogenized in a mixed solution containing $1.67 \%(\mathrm{w} / \mathrm{v})$ metaphosphate, $0.2 \%(\mathrm{w} / \mathrm{v})$ EDTA and $30 \%(\mathrm{w} / \mathrm{v}) \mathrm{NaCl}$, and the homogenates were centrifuged at $5,000 \times g$ for $10 \mathrm{~min}$. Glutathione was determined with deproteinized supernatant according to the method of Ellman(11), using 5,5'-dithiobis-2-nitrobenzoic acid. The total amount of glutathione in reduced and oxidized forms was determined by the enzymic method of Tietze(12), after reduction of glutathione (oxidized form) with glutathione reductase.

A portion of the livers $(0.1$ to $0.2 \mathrm{~g})$ was homogenized in $10 \mathrm{ml}$ of $0.05 \mathrm{~N} \mathrm{HCl}$ solution. To $3 \mathrm{ml}$ of the homogenate was added $1 \mathrm{ml}$ of $0.67 \%(\mathrm{w} / \mathrm{v})$ of thiobarbituric acid, and the mixture was heated at $95^{\circ} \mathrm{C}$ for $30 \mathrm{~min}$. After cooling, $4 \mathrm{ml}$ of $n$ butanol containing $15 \%$ (v/v) methanol was added, and the colored substance was extracted into the butanol layer by shaking. The absorbance was determined at $535 \mathrm{~nm}$, and lipid peroxide was expressed as nmol of malondialdehyde. Lipid peroxides in the serum were determined as described in the previous paper (1), using $0.3 \mathrm{ml}$ of the serum separated from the blood of carotid artery.

Vol. 29, No. 3, 1983 
Protein was determined by the method of Lowry et al.(13), using bovine serum albumin $\mathrm{V}$ as a standard.

Glutathione reductase, glutathione in reduced and oxidized forms, NADPH, NADP, and glucose 6-phosphate were purchased from Böhringer Mannheim $\mathrm{GmBH}$. Other chemicals were of analytical grade.

\section{RESULTS}

\section{Lipid peroxides}

The growth of rats was obviously restricted by feeding the riboflavin-deficient diet, but was not affected by selenium supplement in either case of riboflavindeficient or supplemented diet as shown in Table 1. Content of lipid peroxides increased in the livers and serum of riboflavin-deficient rats, compared with respective value of riboflavin supplemented rats, regardless of selenium supplement in the former rats. Thus, no amelioration in the levels of lipid peroxides was brought about by selenium supplement under these experimental conditions (Table 1).

\section{Glutathione content and enzymic activities}

The glutathione content in the livers was decreased to $3.9 \mu \mathrm{mol} / \mathrm{g}$ liver by feeding rats the riboflavin-deficient diet, compared with a riboflavin supplemented control value of $7.0 \mu \mathrm{mol} / \mathrm{g}$ liver (Table 1). On the other hand, selenium supplement had no effect on the glutathione content of both groups of riboflavin-deficient and supplemented rats. Considerable decrease in glutathione reductase activity was observed in the livers of riboflavin-deficient rats, but no difference was shown by selenium supplement between the riboflavin-deficient and supplemented rat groups. It was remarkable that without selenium supplement, glutathione peroxidase activity of the riboflavin-deficient rats was nearly twice as high as that of supplemented control, in spite of the increase of lipid peroxides in the former. The activity of glutathione peroxidase was remarkably increased by selenium supplement to either group, riboflavin-deficient and supplemented diets, and the activity was nearly the same in the livers of both groups (Table 2).

Decrease of glucose 6-phosphate dehydrogenase activity was brought about by riboflavin deficiency. Contrary to the case of glutathione reductase, selenium supplement decreased the enzymic activity in both groups of riboflavin-deficient and supplemented rats.

\section{Injection of riboflavin to riboflavin-deficient rats}

Twenty weanling rats had been fed the riboflavin-deficient diet for 5 weeks, without selenium supplement, and each of 5 rats was injected riboflavin daily before the time of experiment. The contents of glutathione and lipid peroxides, and the enzymic activities in the livers were determined 24, 48, 72 and $96 \mathrm{~h}$ after the injection, respectively. The glutathione content and the reductase activity recovered to the control levels of riboflavin supplemented rats in $24 \mathrm{~h}$, respectively (Fig. 1). 


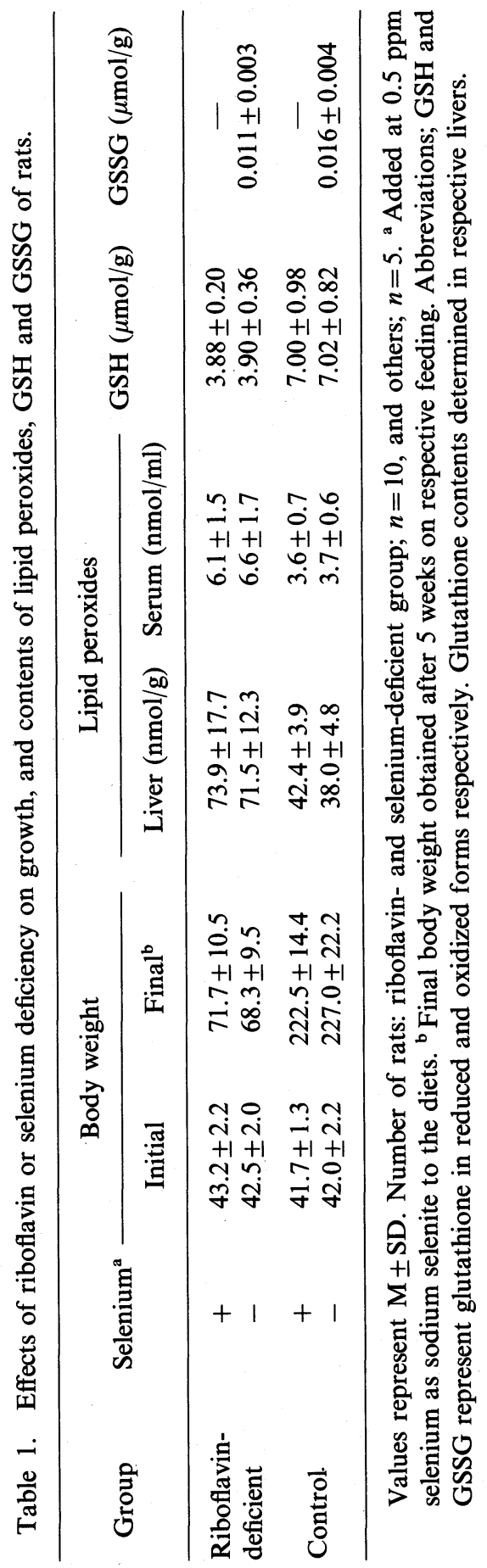

Vol. 29, No. 3, 1983 
Table 2. Changes of glutathione relating enzyme activities of livers of rats fed riboflavin- or selenium-deficient diets.

\begin{tabular}{lcccc}
\hline \multicolumn{1}{c}{ Group } & Selenium & GSH reductase & GSH peroxidase & $\begin{array}{c}\text { Glucose 6-phosphate } \\
\text { dehydrogenase }\end{array}$ \\
\hline Riboflavin- & + & $34 \pm 1$ & $509 \pm 98^{* *}$ & $24 \pm 4^{*}$ \\
deficient & - & $31 \pm 5$ & $213 \pm 14$ & $31 \pm 5$ \\
& + & $56 \pm 5$ & $563 \pm 56^{* *}$ & $38 \pm 3^{* *}$ \\
Control & - & $51 \pm 4$ & $125 \pm 17$ & $48 \pm 2$ \\
\hline
\end{tabular}

Values represent $\mathrm{M} \pm \mathrm{SD}$ of respective enzymic activities (mU/mg protein). Numbers and group of rats are the same shown in Table $1 .^{*}$ and $* * p<0.05$ and $<0.01$ respectively, comparing with selenium-deficient.

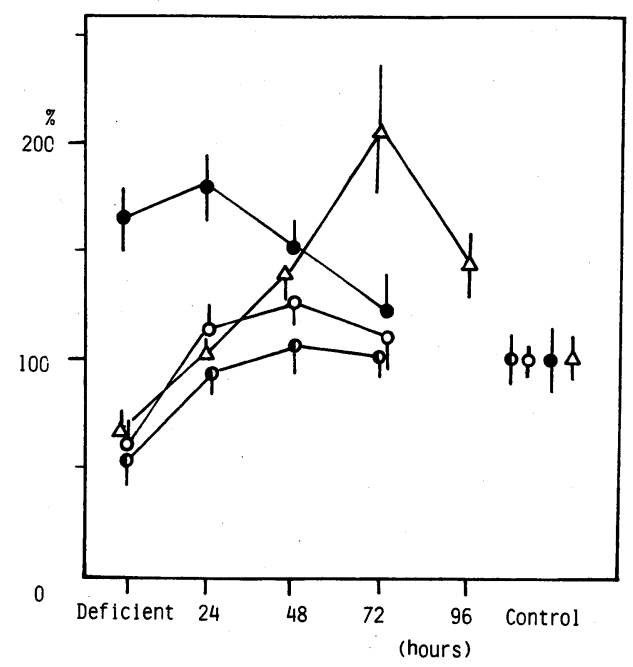

Fig. 1. Effects of riboflavin-supplement on glutathione and enzymic activities related with glutathione of riboflavin-deficient rat livers. Points represent mean values of glutathione $(\circlearrowleft)$, glutathione reductase $(O)$, glutathione peroxidase $(\bullet)$, and glucose 6-phosphate dehydrogenase $(\triangle)$ activities for 5 rats, with their standard deviations represented by vertical bars, and are shown as percent of respective controls (Tables 1 and 2). Time indicates the hours after injection of riboflavin.

The lipid peroxide content in the livers recovered to the control level in $48 \mathrm{~h}$, while that in the serum recovered in $72 \mathrm{~h}$ (livers; $p>0.05$ in $24 \mathrm{~h}$, and serum; $p>0.05$ in $48 \mathrm{~h}$, respectively) (Fig. 2). Without selenium supplement, the glutathione peroxidase activity of riboflavin-deficient rat livers was higher than that of the control, and did not decrease to the control level in $48 \mathrm{~h}(p>0.05)$.

The activity of glucose 6-phosphate dehydrogenase of riboflavin-deficient rats increased twice as high as that of control rats $72 \mathrm{~h}$ after the injection of riboflavin, 


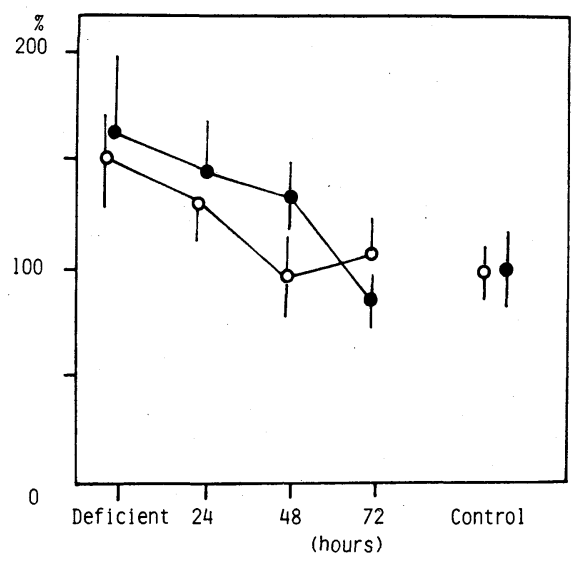

Fig. 2. Changes of lipid peroxide contents by supplement of riboflavin. Points represent mean values of lipid peroxides assayed as malondialdehyde in the livers $(O)$ and serum (๑) of 5 rats, with their standard deviations represented by vertical bars. The values are shown as percent of respective controls. Time indicates the hours after injection of riboflavin.

then a decreasing trend to the normal level of control rats was seen as shown in Fig. 1.

\section{DISCUSSION}

The following evidences indicate that glutathione peroxidase appears to function in preventing lipid peroxidation; (i) increase of lipid peroxidation as monitored by ethane evolution in selenium-deficient rats (14), and (ii) suppression of NADPH-dependent microsomal or mitochondrial lipid peroxidation by addition of glutathione peroxidase $(15,16)$.

In this experiment, remarkable decrease of glutathione peroxidase activity was observed in the livers of rats which were fed diets supplemented with riboflavin but without selenium. On the other hand, the lipid peroxide content in the livers did not differ from that of the rats which were fed the riboflavin and selenium supplemented diet. Glutathione peroxidase activity of the former rat group was $125 \mathrm{mU} / \mathrm{mg}$ protein, and a considerable selenium deficiency seems to have been made in our experiment, compared with the value of $314 \mathrm{mU} / \mathrm{mg}$ protein obtained by Lowrence and Burk from the rats which were fed a selenium-deficient diet for 2 weeks (17). Glutathione peroxidase activity of the riboflavin-deficient rats without selenium was $213 \mathrm{mU} / \mathrm{mg}$ protein, as shown in Table 2. The decrease in the activity was significantly smaller than that of the control, riboflavin supplemented diet group, regardless of increase of lipid peroxides in the riboflavin-deficient rats. It seemed that a small decrease in the activity was due to the restricted growth of riboflavindeficient rats. Therefore, more selenium in the bodies after birth may have been 
retained than in the case of control rats of riboflavin sufficient.

Lowrence and Burk reported that liver glutathione peroxidase activity of the rats which were fed a selenium-deficient diet for 2 weeks decreased from 748 and 318 $\mathrm{mU} / \mathrm{mg}$ protein, using cumene hydroperoxide and $\mathrm{H}_{2} \mathrm{O}_{2}$ as the substrate, respectively (17). In this experiment, glutathione peroxidase activity was not determined with $\mathrm{H}_{2} \mathrm{O}_{2}$ as the substrate, but a comparable decrease in the activity with their results was obtained with the former substrate to which selenium-dependent and -independent enzyme could react. Since the rats which were fed seleniumdeficient and riboflavin supplemented diet did not accumulate lipid peroxides in the livers, it may be suggested that the requirement of selenium-dependent enzyme to protect against lipid peroxidation is less than that of those rat livers of the seleniumdeficient group or a considerable decrease in selenium-independent glutathione peroxidase activity had been brought about in the riboflavin-deficient rats. Using dinitrochlorobenzene as a substrate, a slight decrease in glutathione transferase activity was observed in the riboflavin-deficient rat livers, but not in those of selenium-deficient (data not shown). Therefore, it will be necessary to study the role of selenium-independent enzyme as a protecting mechanism against lipid peroxidation in riboflavin-deficient rats.

In vitro experiment, the velocity of glutathione peroxidase was shown to be in proportion with the concentration of glutathione in the case when it fell to less than $1 \mathrm{~mm}$ (about one-tenth of the usual level) (18). Our findings together with these observations indicate that the protecting mechanism against lipid peroxidation in the riboflavin-deficient rats was deteriorated by a decrease in glutathione content rather than by that of glutathione peroxidase activity at least with respect to the selenium-dependent enzyme.

Tateishi et al. suggested the factors that affect glutathione content in rat livers as the following: (i) synthesizing enzymes (glutathione synthetase and $\gamma$-glutamylsynthetase), (ii) availability of glutamine, cysteine and glycine, and (iii) degradating enzyme ( $\gamma$-glutamyltransferase) (19). They observed that glutathione content in the rat livers decreased between two-thirds and half of the normal level on starvation, and it rapidly recovered to normal level by feeding. They suggested that the level of glutathione was dependent on food intake, and found that it was regulated by the degradative enzyme activity. In riboflavin-deficient rat livers, the content of total glutathione in reduced and oxidized forms decreased to $55 \%$ of the control level of riboflavin supplemented rats, and that of oxidized form did not increase but slightly decreased. Therefore, the cause of the decrease in content of reduced form of glutathione in riboflavin-deficient rats can not be attributed only to the decrease of glutathione reductase activity, but to insufficiency of availability of amino acids which may have resulted from the restricted growth or food intake as in the case of fasted rats mentioned above.

Decrease in glutathione reductase activity may restrain the availability of glutathione in reduced form in a particular location in the cell and it probably affects lipid peroxidation through glutathione peroxidase. Meredith and Reed 
demonstrated two pools of glutathione in hepatocytes, and that about $85 \%$ of cellular total glutathione was in a cytoplasmic location, and a small pool of the rest in the mitochondria (20). Though distribution of glutathione in hepatocytes was not separately determined in our experiment, shortage of glutathione content and decrease in glutathione reductase activity were presumed to occur in the liver mitochondria of riboflavin-deficient rats, as in the case of whole livers, and these may have caused the deterioration in the protecting mechanism against lipid peroxidation in mitochondria. Hence decrease in activity of NADH-cytochrome $c$ reductase in liver mitochondria which is rotenone insensitive and distributed in outer membrane was slight in riboflavin-deficient rats, compared with that of other flavoenzyme activities (unpublished data), and NADH-dependent lipid peroxidation with mitochondria was accerelated by addition of rotenone in vitro $(21,22)$, lipid peroxidation in particular organella as mitochondria may be accelerated by riboflavin deficiency as a consequence of the decrease in glutathione reductase activity together with the decrease in glutathione concentration.

Low glucose 6-phosphate dehydrogenase activity seems to restrict reduction of oxidized form of glutathione through regeneration of NADP, and it may have accelerated lipid peroxidation in riboflavin-deficient rats, together with low glutathione reductase activity.

\section{REFERENCES}

1) Taniguchi, M. (1980): Effects of riboflavin deficiency on lipid peroxidation of rat liver microsomes. J. Nutr. Sci. Vitaminol., 26, 401-413.

2) Pederson, T. C., Buege, J. A., and Aust, S. D. (1973): Microsomal electron transport. The role of reduced nicotinamide adenine dinucleotide phosphate-cytochrome $c$ reductase in liver microsomal lipid peroxidation. J. Biol. Chem., 248, 7134-7141.

3) Hirokata, Y., Shigematsu, A., and Omura, T. (1978): Microsomal electron transport. Immunochemical study on the pathway of electron flow in reduced nicotinamide adenine dinucleotide-dependent microsomal lipid peroxidation. J. Biochem., 83, 431440.

4) Svingen, B. A., Buege, J. A., O’Neal, F. O., and Aust, S. D. (1979): The mechanism of NADPH-dependent lipid peroxidation. The propagation of lipid peroxidation. J. Biol. Chem., 254, 5892-5899.

5) Hara, T., and Taniguchi, M. (1982): Abnormal NADPH-cytochrome P-450 reductase in the liver microsomes of riboflavin-deficient rats. Biochem. Biophys. Res. Commun., 104, 394-401.

6) Vermilion, J. L., and Coon, M. J. (1978): Identification of the high and low potential flavins of liver microsomal NADPH-cytochrome P-450 reductase. J. Biol. Chem., 253, 8812-8819.

7) Little, C., and O'Brien, P. J. (1968): An intracellular GSH-peroxidase with a lipid peroxide substrate. Biochem. Biophys. Res. Commun., 31, 145-150.

8) Olson, O. E., Palmer, I. S., and Whitehead, E. I. (1973): Determination of selenium in biological materials, in Methods of Biochemical Analysis, Vol. 21, ed. by Glick, D., Interscience Publ., New York, pp. 39-78.

9) Tappel, A. L. (1978): Glutathione peroxidase and hydroperoxides, in Methods in

Vol. 29, No. 3, 1983 
Enzymology, Vol. 52, Part c, ed. by Fleischer, S., and Packer, L., Academic Press, New York, pp. 506-513.

10) Carlberg, I., and Mannervik, B. (1975): Purification and characterization of the flavoenzyme glutathione reductase from rat liver. J. Biol. Chem., 250, 5475-5480.

11) Ellman, G. L. (1959): Tissue sulfhydryl groups. Arch. Biochem. Biophys., 82, 70-77.

12) Tietze, F. (1969): Enzymic method for quantitative determination of nanogram amounts of total and oxidized glutathione. Anal. Biochem., 27, 502-522.

13) Lowry, O. H., Rosebrough, N. J., Farr, A. L., and Randall, R. J. (1951): Protein measurement with the Folin phenol reagent. J. Biol. Chem., 193, 265-275.

14) Hafeman, D. G., and Hoekstra, W. G. (1977): Lipid peroxidation in vivo during vitamin $\mathrm{E}$ and selenium deficiency in the rats as monitored by ethane evolution. J. Nutr., 107, 666-672.

15) McCay, P. B., Gibson, D. D., Fong, K.-L., and Hornbrook, K. R. (1976): Effect of glutathione peroxidase activity on lipid peroxidation in biological membranes. Biochem. Biophys. Acta, 431, 459-468.

16) Burk, R. F., Trumble, M. J., and Lawrence, R. A. (1980): Rat hepatic cytosolic glutathione-dependent enzyme protection against lipid peroxidation in the NADPHmicrosomal lipid peroxidation system. Biochem. Biophys. Acta, 618, 35-41.

17) Lawrence, R. A., and Burk, R. F. (1976): Glutathione peroxidase activity in seleniumdeficient rat liver. Biochem. Biophys. Res. Commun., 71, 952-958.

18) Flohe, L. (1979): Glutathione peroxidase: fact and function, in Oxygen Free Radicals and Tissue Damage, Ciba Foundation Symposium 65, Excerpta Medica, Amsterdam, Oxford, New York, pp. 102-103.

19) Tateishi, N., Higashi, T., Naruse, A., Nakashima, K., Shiozaki, H., and Sakamoto, Y. (1977): Rat liver glutathione: Possible role as a reservoir of cysteine. J. Nutr., 107, 51-60.

20) Meredith, M. J., and Reed, D. J. (1982): Status of the mitochondrial pool of glutathione in the isolated hepatocyte. J. Biol. Chem., 257, 3747-3753.

21) Taniguchi, M. (1979): NADH-dependent lipid peroxidation by liver mitochondria. Nakamura Gakuen Kenkyu Kiyo (in Japanese), 12, 165-168.

22) Takayanagi, R., Takeshige, K., and Minakami, S. (1980): NADH- and NADPHdependent lipid peroxidation in bovine heart submitochondrial particles. Biochem. J., 192, 853-860. 